\title{
Do aspirating systems have a role in preventing COVID-19 transmission among dental healthcare workers?
}

\section{Neeraj Gugnani*1 and Shalini Gugnani ${ }^{2}$}

\section{A commentary on}

\section{Sarapultseva M, Hu D, Sarapultsev A.}

SARS-CoV-2 Seropositivity among Dental Staff and the Role of Aspirating Systems. JDR Clin Trans Res 2021; 6: 132-138.

\section{Abstract}

Aim The aim of this retrospective cohort study was to determine and compare the seropositivity rates of SARS-CoV- 2 among dental healthcare workers (HCWs) working in three different clinics using different types of aspirating systems. The study took place in Ekaterinburg (Russian Federation).

Methods A total of $157 \mathrm{HCWs}$ from three different clinics (56, 60 and $41 \mathrm{HCWs}$, respectively) who worked during the COVID-19 pandemic period (May to August 2020) constituted the cohort. All of the three chosen clinics had followed COVID-19 screening, triage and other recommendations for safe practice. In addition to using personal protective equipment and other common barrier methods to lower virus transmission, these clinics were equipped with different types of aspirating systems that included V6000 used in dry mode, V6000 used in semi-dry mode and VS900, respectively. All HCWs underwent serological testing once a week to detect immunoglobulin $G$ and $M$ antibodies against SARS-CoV-2 using SARS-CoV-2-IgG-EIA-BEST and SARS-CoV-2-IgM-EIA-BEST enzyme immunoassay kits (Vector-Best). Results An overall prevalence of seropositivity was observed to be $11.5 \%(19 / 157 \mathrm{HCWs})$ over a five-month follow-up. The prevalence of infection was not found to be associated with sex or the role of the member in the dental team (dentist/dental assistant). Significantly higher infection rates $(p<0.001)$ were observed among HCWs working in the clinic equipped with the VS900 aspirating vacuum pump without HEPA filters, while the lowest infection rate was found among HCWs working in the clinic using the V6000 aspirating system in dry mode. Conclusions HCWs working in the clinic equipped with an aspirating system which has HEPA filters and released the air into an external environment (V6000) exhibited significantly lower seroprevalence rates compared to HCWs in the clinic using an aspirating system without HEPA filters which released air within the dental operatory away from the operation site (VS900).

\section{Commentary}

Dental office personnel are considered to be at high risk for COVID-19 transmission owing to their occupational exposure via bio-aerosols and through indirect contact with surfaces that might have been contaminated by respiratory droplets from patients. ${ }^{1}$ In

\section{GRADE rating}

Practice point

- Use of an aspirating system with HEPA filters and an in-built mechanism for air evacuations outside the clinical environment is useful in minimising the spread of COVID-19 in the dental clinic and hence clinicians should consider adopting such systems for a safe dental practice.

fact, production of aerosols and splatter during dental procedures has always been considered a health concern as these may spread infections among dental healthcare workers (HCWs) and other patients. $^{2}$

There are many methods documented in the literature that can be used to contain the spread of these aerosols, ${ }^{3}$ but routinely, dental professionals depend mainly on the use of personal protective techniques and other barriers (for example, use of rubber dam, saliva ejectors, suction) in their day-to-day clinical practice to avoid cross contamination. However, in the light of the current pandemic, it has become imperative to collect, collate and translate available evidence regarding the efficacy of 'all' the possible strategies that can help to curb the spread of aerosol-associated COVID-19 in dental clinics. Based on published literature and various recommendations, a recent Cochrane review has suggested a set of six-layer measures that can be used for infection control in a dental office. These include: use of personal protective barriers; interventions that can prevent contamination of aerosols in the oral cavity (for example, use of mouthwash); interventions that can prevent aerosols escaping from the mouth (for example, use of rubber dam); interventions that may prevent the spread of aerosols away from the operating site and thereby reduce the overall concentration of aerosols in the dental operatory (for example, use of high-volume suction); and lastly interventions that can decontaminate the aerosols in the atmosphere (for example, use of UV light etc). ${ }^{2}$ The authors of the Cochrane review indicated that due to the paucity of robust evidence, reliable conclusions could not be drawn in favour of any of the systems to limit aerosol transmission. However, in order to evaluate the efficacy of highvolume evacuation (HVE) systems, the authors of the Cochrane review conducted a meta-analysis and found these to be beneficial in reducing the level of aerosol contamination when compared to no HVE systems; however, there is still a need to determine and compare the efficacy of different commercially available aspirating systems in controlling COVID-19 transmission. ${ }^{3}$

The current paper is an attempt to evaluate the effectiveness of three different aspirating systems used in dental clinics during the COVID-19 pandemic. During the pandemic, it is, of course, difficult and ethically unjustifiable to design randomised controlled trials (RCTs) to evaluate the risk of transmission of the disease. Hence, any data collected over time or in a retrospective manner adds to our understanding of the disease and its 
behaviour. Contextually, this study was conducted in the peak of the pandemic period between May-August 2020, for which the authors have aptly chosen a retrospective cohort design. This research is indeed much needed at present and the results of studies that aim to determine the efficacy of various strategies to curb the spread of the virus are essential. The second/third wave of the pandemic is still rife in various countries, and many countries are reinstating lockdown and are only allowing limited mobility in order to curb the spread of the virus. In such circumstances, it is crucial that we safeguard our community of dental HCWs by providing them with evidence-based safety guidelines.

The aim of this retrospective cohort study was to assess the SARS-CoV-2 seropositivity among dental HCWs working in three different clinics (two private clinics and one government centre) in Ekaterinburg (Russian Federation) which have used three different aspirating systems. The study included 157 HCWs who had direct contacts with the patients. The mean age of the included participants was $43.58 \pm 1.66$ years. Among the 157 included HCWs, 119 were women; additionally, 50\% were dentists and $50 \%$ included other dental HCWs. HCWs who are $>65$ years old, have other chronic diseases, have immunological concerns or are pregnant are not permitted to work according to Russian Federation guidelines, and hence were not part of the cohort. In general, all safety protocols required to avoid or minimise the contact between HCWs and patients were followed at all three clinics. These included teleconsultation, screening of patients regarding their travel history, contact tracing, triage examination to determine any history of fever or respiratory illness etc. Patients with any suspicion of the disease were advised to quarantine for 14 days and their definitive dental treatment was deferred until after this time. Treatment was also restricted to emergency and urgent cases. The clinics that were chosen for this retrospective study were similar in surface area (not less than $14 \mathrm{~m}^{2}$ ) but used three different aspirating systems - two clinics used the aspirating central vacuum pump V6000 (Dürr Dental AG) in dry mode and in semi-dry mode, respectively, and the third clinic used the aspirating vacuum pump VS900 (Dürr Dental $A G)$. In general, suction devices used in dentistry are fitted with an evacuation system that can suck in and eject large volumes of air at high speed. Many different commercial systems are available that exhibit different capacities - some have additional features; for example, HEPA or carbon filters. ${ }^{4}$ The basic difference between the V6000 and VS900 is that the former is a central aspiration machine with a vacuum controller and a H14 HEPA bacterial filter, and which releases the collected air into an 'external' area outside the clinic, while the VS900 is an aspirating vacuum pump without a HEPA filter. Moreover, the VS900 collects the air and releases it in the same way, away from the operation site but within the clinical area.

All HCWs underwent serological testing once a week to detect immunoglobulin $\mathrm{G}$ and $\mathrm{M}$ antibodies against SARS-CoV-2. Over the said five-month follow-up period, the overall prevalence of SARSCoV-2 infection in the chosen cohort was observed to be $11.5 \%$ (19/157 HCWs were found to be seropositive). Pearson's correlation coefficient with Yate's correction was used for data analysis. The prevalence rate did not exhibit any association with sex or the role of the member in the dental team (dentist or dental assistant). The number of HCWs exhibiting seropositivity for SARS-CoV-2 was found to be significantly higher among HCWs who were using the
VS900 (15/41 HCWs showed presence of antibodies) compared to HCWs working in the other two clinics using the V6000, with the lowest infection rate found among HCWs working in the clinic using the V6000 in dry mode (only $1 / 56$ for dry mode and 3/60 for semi-dry mode). These notable differences have been rightly explained owing to the fact that the VS900 system releases the air within the dental operatory, which mimics the natural route of aerosol transmission. Patients' respiratory aerosols may remain suspended within the clinical environment for a long period of time and may lead to the transfer of contagion among HCWs. On the other hand, the V6000 discharges the collected air into the external environment, with no contagion being released in the clinic area and thus decreasing the probability of contact transmission. This might have been augmented by the presence of HEPA filters in the V6000 model compared to no HEPA filters in the VS900 model.

Further, the lowest rates with the V6000 used in dry mode have been explained on the basis that in 'dry' suction systems, the aspirated fluids get separated from the air at every treatment unit, whereas in 'semi-dry' suction systems, this separation of fluids and air occurs in a central unit connected to multiple treatment units. Conclusively, aspirating systems fitted with HEPA filters, and which release the collected air outside the clinical environment, are helpful in stopping aerosol-based contagion transmission. As this was a retrospective study, long-term prospective studies (prospective cohort and RCTs) including diverse cohorts should be done to substantiate these findings.

Overall, the rate of seropositivity in the chosen cohort was observed to be higher than the general population residing in other parts of the Russian Federation, which clearly warrants the need to recognise the impending threat from these aerosols and to minimise these to the greatest possible extent. Keeping an optimistic view, the literature suggests that with ongoing vaccination, the pandemic will come to an end but the disease may still remain endemic in society. ${ }^{5}$ The associated morbidity with the disease will therefore certainly require us to modify our dental practices and hence the use of very high-quality aspirating systems may become a necessary component of armamentarium in dental clinics worldwide.

\section{Author affiliations \\ ${ }^{1}$ Department of Paedodontics and Preventive Dentistry, DAV (C) Dental College Yamunanagar, Haryana, India; ${ }^{2}$ Department of Periodontics and Oral Implantology, DAV (C) Dental College Yamunanagar, Haryana, India. \\ *Correspondence to: Neeraj Gugnani}

\section{References}

1. Gugnani N, Gugnani S. Safety protocols for dental practices in the COVID-19 era. Evid Based Dent 2020; 21: 56-57.

2. Kumbargere Nagraj S, Eachempati P, Paisi M, Nasser M, Sivaramakrishnan G, Verbeek J H. Interventions to reduce contaminated aerosols produced during dental procedures for preventing infectious diseases. Cochrane Database Syst Rev 2020; DOI: 10.1002/14651858.CD013686.pub2.

3. Harrel S K, Molinari J. Aerosols and splatter in dentistry: a brief review of the literature and infection control implications. J Am Dent Assoc 2004; 135: 429-437.

4. Avasthi A. High volume evacuator (HVE) in reducing aerosol - an exploration worth by clinicians. I Dent Health Oral Disord Ther 2018; 9: 165-166.

5. Veldhoen M, Simas J P. Endemic SARS CoV 2 will maintain post-pandemic immunity. Nat Rev Immunol 2021; 21: 131-132.

Evidence-Based Dentistry (2021) 22, 50-51.

https://doi.org/ 10.1038/s41432-021-0171-5 